\title{
LIGHT SCATTERING BY TRAPPED THREE-LEVEL ATOMS
}

\author{
P. KREKora AND J. Mostowski \\ Institute of Physics, Polish Academy of Sciences \\ Al. Lotników 32, 02-668 Warszawa, Poland \\ (Received July 11, 1994; in final form October 12, 1994)
}

\begin{abstract}
We study the scattering of the probe light beam by randomly distributed strongly pumped atoms in a pencil-like medium. The density of atoms is assumed to be small (of the order of one atom per light wavelength), therefore the medium formed by the atoms cannot be treated as a continuous one. Such a configuration of atoms can be practically realized in atomic traps. We show that intensity of the scattered light increases with the third power of the probe beam intensity and with the second power of the number of atoms. We also show that under certain conditions the light is scattered into few cones.
\end{abstract}

PACS numbers: $42.50 . F x, 42.50 . G y$

\section{Introduction}

The possibility to isolate a single particle in a small region of space has initialized a great number of experimental and theoretical papers dealing with problems of the atom trap [1-3]. Single particle at rest is a fascinating tool for testing the interaction of light with matter on the most elementary level.

Experimental techniques allow one to isolate and cool by light pressure force not only one atom but arbitrary number of atoms, and what is most important, to control the number of them. We would like to show in this paper that the system of many trapped atoms is interesting from the theoretical point of view.

The most convenient method of studying the properties of an atom cloud is light scattering.

In this paper we will study light scattering from one-dimensional atom cloud and we will treat the system as a discrete medium. The reason is that the typical distance between atoms is of the order of the optical wavelength. Moreover, we will study interaction between the fields and the atoms in semi-classical picture.

Our medium consists of three-level atoms interacting with two external waves (see Fig. 1a) and the system forms pencil-shaped medium. One of the waves is 
a)

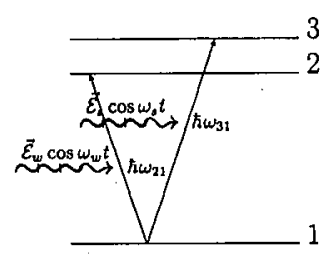

b)

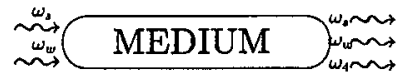

c)

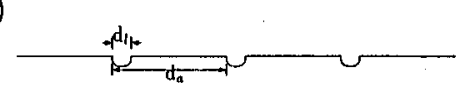

Fig. 1. (a) Model of the atom interacting with two external fields. (b) Scheme of the problem, $\omega_{s}$ and $\omega_{w}$ are the external waves, $\omega_{4}$ is the scattered light that we observe. (c) The figure shows the scheme of the atom trap potential we assume. $d_{a}$ is the average distance between atoms, $d_{l}$ is the localization length.

strong (pumping) wave and prepares atom dipoles, and the second one is weak (probing) wave. We will assume that the external waves are plane waves propagating along the axis of the medium and they have the same linear polarization. We will find the equations governing the atom evolution and later on we will solve them numerically under steady-state limit. Having atoms' states we will find the radiation of the system in far field. Because atoms are distributed randomly in the neighborhood of their equilibrium positions, the electric field of the scattered light has to be averaged with respect to the positions of atom.

Our paper is organized as follows. In Sec. 2 we describe the model of the medium. Later on we formulate the Hamiltonian of the system in Sec. 3 followed in Sec. 4 by the derivation of the equations for the atomic density matrix. We solve these equations numerically and results are presented in Sec. 5 . We summarize in Sec. 6.

\section{Description of the medium}

We assume a very simplified model of ork-dimensional trap, and we assume that the mechanism of trapping does not disturb the processes we examine. Atoms are distributed on a line around the equilibrium positions separated by some constant value $d_{a}$. Atoms can be localized within an interval of length $d_{l}$, we called it "localization length". Figure 1c shows the scheme of the trap we have assumed.

The model of atom trap presented above is a very simplified one, but we do not need to consider it more precisely.

Below we present parameters characterizing our model:

- dumping rate of the 3 rd level $-\Gamma$, 
- wavelength of the probe beam $-\lambda$,

- dumping rate of the 2 nd level $-\Gamma_{w}$, with $\Gamma_{w} / \Gamma \approx 1$,

- pump field Rabi frequency $-\Omega_{s}=d_{13} \mathcal{E}_{s} / \hbar, \Omega_{s} / \Gamma>1$,

- probe field Rabi frequency $-\Omega_{w}=d_{12} \mathcal{E}_{w} / \hbar, \Omega_{w} / \dot{\Gamma}<1, \Omega_{s} / \Omega_{w} \gg 1$,

- pump field detuning $-\Delta_{s}, \Delta_{s} / \Gamma \approx 1$,

- probe field detuning $-\Delta_{w}, \Delta_{w}=-\Omega_{s}$,

- number of atoms $-N \approx 100$,

- average distance between atoms - $d_{a}$, with $d_{a} / \lambda \approx 1$,

- localization length $-d_{l}$, with $d_{l} / d_{a}<0.5$.

The first two parameters are fixed for all computations and we treat them as our units of frequencies and distances respectively. In the above $\mathcal{E}$ denotes amplitude of the classical field, and $d_{\mu \nu}$ - dipole moments.

\section{Formulation of the problem}

The Hamiltonian of the system is given by

$$
\hat{H}=\hat{H}_{\text {at }}+\hat{H}_{\text {field }}+\hat{H}_{\text {int }} \text {, }
$$

with

$$
\begin{aligned}
& \hat{H}_{\mathrm{at}}=\sum_{j=1}^{N} \sum_{\mu=1}^{3} W_{\mu}^{(j)} \hat{\sigma}_{\mu \mu}^{(j)}, \\
& \hat{H}_{\text {field }}=\frac{1}{8 \pi} \int_{V}\left(\boldsymbol{E} \boldsymbol{E}^{*}+\boldsymbol{B} \boldsymbol{B}^{*}\right) \mathrm{d} \boldsymbol{r} \\
& \hat{H}_{\text {int }}=-\sum_{j=1}^{N} \hat{d}^{(j)} \hat{E} .
\end{aligned}
$$

$\hat{H}_{\text {at }}$ is the Hamiltonian of the free three-level atoms forming the medium. $\hat{\sigma}_{\mu \nu}$ are the atomic projection operators in the Heisenberg picture, $W_{\mu}$ are the eigenvalues of the atomic Hamiltonian. In the above Greek letters are used to index the energy levels, while Roman ones denote the atoms. Levels 3 and 2 are the excited states, and level 1 is the ground state. $N$ is the total number of atoms.

$\hat{H}_{\text {field }}$ is the Hamiltonian for the total field and $\boldsymbol{B}=\mu_{0} \boldsymbol{H}$ is the magnetic induction. $\boldsymbol{E}$ and $\boldsymbol{H}$ are respectively the vectors of the electric and magnetic fields of the light.

The electric and magnetic fields $\boldsymbol{E}_{j}$ and $\boldsymbol{H}_{j}[4]$, which act on the $j$-th dipole of the medium can be divided into the external fields $\boldsymbol{E}_{i}, \boldsymbol{H}_{i}(i=s, w)$ (propagating with the vacuum velocity $c$ ) and the contribution arising from all the dipoles

$$
\begin{aligned}
& \boldsymbol{E}_{j}=\boldsymbol{E}_{s}+\boldsymbol{E}_{w}+\sum_{l \neq j} \boldsymbol{E}_{j l}, \\
& \boldsymbol{H}_{j}=\boldsymbol{H}_{s}+\boldsymbol{H}_{w}+\sum_{l \neq j} \boldsymbol{H}_{j l},
\end{aligned}
$$


the summation extending over all the dipoles except the $j$-th one. At the point $\boldsymbol{r}_{j}$ where the $j$-th dipole is situated, the field of the $l$-th dipole is obtained from

$$
\begin{aligned}
& \boldsymbol{E}_{j l}(t)=\frac{1}{4 \pi \varepsilon_{0}} \operatorname{curl} \operatorname{curl} \frac{d_{l}\left(t-R_{j l} / c\right)}{R_{j l}}, \\
& \boldsymbol{H}_{j l}(t)=\frac{1}{4 \pi} \operatorname{curl} \frac{\dot{d}_{l}\left(t-R_{j l} / c\right)}{R_{j l}} .
\end{aligned}
$$

$d_{l}(t)$ denotes the moment of the $l$-th dipole, $R_{j l}=\left|r_{j}-r_{l}\right|$, and the operation curl is taken with respect to the coordinates $x_{j}, y_{j}, z_{j}$ of the $j$-th dipole. As our medium is pencil-shaped one, we assume that the external fields depend only on $z$ coordinate, and using retarded, local time $\tau=t-z / c$ we can write classical fields as follows:

$$
\begin{aligned}
& \left.\begin{array}{c}
\boldsymbol{E}_{s}(r, t)=\overrightarrow{\mathcal{E}}_{s} \mathrm{e}^{-\mathrm{i}\left(\omega_{s} \tau\right)}+\text { c.c. } \\
\boldsymbol{H}_{s}(r, t)=\overrightarrow{\mathcal{H}}_{s} \mathrm{e}^{-\mathrm{i}\left(\omega_{s} \tau\right)}+\text { c.c. }
\end{array}\right\} \text { are the strong (pump) fields, } \omega_{s}=\omega_{31}, \\
& \left.\begin{array}{l}
\boldsymbol{E}_{w}(\boldsymbol{r}, t)=\overrightarrow{\mathcal{E}}_{w} \mathrm{e}^{-\mathrm{i}\left(\omega_{w} \tau\right)}+\text { c.c. } \\
\boldsymbol{H}_{w}(r, t)=\overrightarrow{\mathcal{H}}_{w} \mathrm{e}^{-\mathrm{i}\left(\omega_{w} \tau\right)}+\text { c.c. }
\end{array}\right\} \text { are the weak (probe) fields, } \omega_{s}=\omega_{21} .
\end{aligned}
$$

As we have pointed above we treat the medium as a discrete one so we will not consider the moment of dipole as a continuous function of position. Since the medium is a diluted one we assume that the external waves have constant amplitude over all the medium and we neglect the fields in our consideration.

We describe the interaction Hamiltonian in the following section.

\section{Evolution of the atom states}

Since our problem is to find the scattering by system of atoms, we have to find the radiation of single atom. Thus we need to know the state of the atom placed in fields of two external waves and fields having origin in other atoms. State of the atom is described by the density matrix $\hat{\rho}$. Evolution of this operator in the Schrödinger picture is given by equation

$$
\mathrm{i} \hbar \dot{\hat{\rho}}=[\hat{H}, \hat{\rho}] \text {. }
$$

Next we describe the interaction Hamiltonian $\boldsymbol{H}_{\text {int }}$ between the fields and the atoms. The dipole moments of the atoms in $t=0$ are assumed to be the same irrespectively of location, such that $d_{11}=d_{22}=d_{33}=0$, since we can choose arbitrary phase of atom states and assume $d_{12}=d_{21}, d_{13}=d_{31}, d_{23}=d_{32}=0$. To find the evolution of the elements of the density matrix it is necessary to take average values in respective states.

Taking classical field and assuming the same polarization of both external waves (orthogonal to the propagation direction) we can write average values of Hamiltonian in particular atom states

$$
\begin{aligned}
& \langle 3|\hat{H}| 1\rangle=\langle 1|\hat{H}| 3\rangle=-d_{31} E_{s}, \\
& \langle 2|\hat{H}| 1\rangle=\langle 1|\hat{H}| 2\rangle=-d_{21} E_{w},
\end{aligned}
$$


$\langle 3|\hat{H}| 2\rangle=\langle 2|\hat{H}| 3\rangle=0$,
$\langle 3|\hat{H}| 3\rangle=W_{3}$,
$\langle 2|\hat{H}| 2\rangle=W_{2}$
$\langle 1|\hat{H}| 1\rangle=W_{1}$

Also we have (see Fig. 1a):

$$
\begin{gathered}
\omega_{w} \approx \frac{W_{2}-W_{1}}{\hbar}=\omega_{21}, \\
\omega_{s} \approx \frac{W_{3}-W_{1}}{\hbar}=\omega_{31} .
\end{gathered}
$$

We can count the evolution of elements of the density matrix following the equation:

$$
i \hbar\langle\mu|\dot{\hat{\rho}}| \nu\rangle=\langle\mu|\hat{H} \hat{\rho}-\hat{\rho} \hat{H}| \nu\rangle,
$$

with $\mu$ and $\nu$ running across all the atom states 1,2,3. Extending the right side we can write

$$
i \hbar\langle\mu|\dot{\hat{\rho}}| \nu\rangle=\sum_{\xi=1}^{3}(\langle\mu|\hat{H}| \xi\rangle\langle\xi|\hat{\rho}| \nu\rangle+\langle\mu|\hat{\rho}| \xi\rangle\langle\xi|\hat{H}| \nu\rangle) .
$$

For one atom we can write a matrix equation given under rotation wave approximation (RWA) and after adding fenomenological dumping constants

$$
\dot{i} \dot{\varrho}=\mathcal{M} \varrho+\varrho_{n}
$$

with

$$
\begin{aligned}
& \varrho=\left[\varrho_{12}, \varrho_{21}, \varrho_{13}, \varrho_{31}, \varrho_{23}, \varrho_{32}, \varrho_{11}, \varrho_{22}\right], \\
& \varrho_{n}=\left[0,0,-\frac{\Omega_{s}}{2}, \frac{\Omega_{s}}{2}, 0,0, \mathrm{i} \Gamma, 0\right],
\end{aligned}
$$

$\varrho_{n}$ arises after eliminating $\varrho_{33}\left(\varrho_{11}+\varrho_{22}+\varrho_{33}=1\right)$.

We can write

$$
\mathcal{M}=\mathcal{S}+\mathcal{W} \text {. }
$$

The diagonal part of $\mathcal{S}$ describes detunings and dumping, and all non-diagonal, non-zero elements of that matrix are proportional to $\Omega_{s} . \mathcal{W}$ is a non-diagonal matrix and all non-zero elements are proportional to $\Omega_{w}$.

We assumed the dipole decay rate to be two times smaller than the inversion damping rate (just like for a spontaneous emission).

For $N_{t}$ atoms the size of the matrix $\mathcal{M}$ rises to $8 N_{t} \times 8 N_{t}$ because of the interaction between atoms forming medium (through radiation and re-absorption of the light) and it forms a block matrix as we show below

$$
\mathcal{M}^{(8 N \times 8 N)}=\left(\begin{array}{cccc}
\mathcal{M}^{(8 \times 8)} & \mathcal{C}_{12}^{(8 \times 8)} & \ldots & \mathcal{C}_{1 N_{t}}^{(8 \times 8)} \\
\mathcal{C}_{21}^{(8 \times 8)} & \mathcal{M}^{(8 \times 8)} & \ldots & \mathcal{C}_{2 N_{t}}^{(8 \times 8)} \\
\vdots & \vdots & \vdots & \vdots \\
\mathcal{C}_{N 1}^{(8 \times 8)} & \mathcal{C}_{N 2}^{(8 \times 8)} & \ldots & \mathcal{M}^{(8 N \times 8 N)}
\end{array}\right)
$$


with

$$
\mathcal{M}^{(8 \times 8)}=\mathcal{S}^{(8 \times 8)}+\mathcal{W}^{(8 \times 8)}
$$

and all the non-zero elements of matrix $\mathcal{W}^{(8 \times 8)}$ are proportional to $\Omega_{w} \cdot \mathcal{C}_{i j}^{(8 \times 8)}$ describe an atom-atom interaction.

Since all the non-zero and non-diagonal elements of each matrix obey the conditions

$$
s_{i j} \gg w_{i j}, \quad s_{i j} \gg c_{i j},
$$

we can look for solution of Eq. (1) (in the steady-state limit) as a series expansion in $\left(E_{w}+\sum E_{j}\right) / E_{s}$ :

- zero approximation:

$$
\mathcal{S}^{(8 N \times 8 N)} \varrho_{0}^{8 N}+\varrho_{n}^{8 N}=0
$$

- first approximation:

$$
\mathcal{S}^{(8 N \times 8 N)} \varrho_{1}^{8 N}+\left(\mathcal{W}^{(8 N \times 8 N)}+\mathcal{C}^{(8 N \times 8 N)}\right) \varrho_{0}^{8 N}=0,
$$

- second approximation:

$$
\mathcal{S}^{(8 N \times 8 N)} \varrho_{2}^{8 N}+\left(\mathcal{W}^{(8 N \times 8 N)}+\mathcal{C}^{(8 N \times 8 N)}\right) \varrho_{1}^{8 N}=0,
$$

- third approximation:

$$
\mathcal{S}^{(8 N \times 8 N)} \varrho_{3}^{8 N}+\left(\mathcal{W}^{(8 N \times 8 N)}+\mathcal{C}^{(8 N \times 8 N)}\right) \varrho_{2}^{8 N}=0
$$

Since the atom-atom interaction appears in the third order of approximation, we expand $\varrho$ to the third order. The knowledge of the density matrix elements allows us to compute radiation of atoms and the whole system. For the far field we have

$$
\mathcal{E}(r) \sim \sum_{j=1}^{N_{t}} d_{21}\left(\varrho_{21}^{(j)} \mathrm{e}^{-\mathrm{i} k r_{n}}-\varrho_{12}^{(j)} \mathrm{e}^{\mathrm{i} k r_{n}}\right),
$$

with $\left(\varrho_{21}^{(j)}\right)^{*}=\varrho_{12}^{(j)}=f\left(\varrho_{12}^{(i \neq j)}, \varrho_{21}^{(i \neq j)}\right)$. This is computed numerically.

\section{Results}

In our computations we examined intensity of the scattered light as a function of the direction of scattering. We called the intensity of scattered light in the forward direction as "forward scattered light intensity" and analogously for backward direction. Moreover we integrated the intensity around the medium in the plane containing the axis of the medium and perpendicular to the vector of the light polarization. We called that value "total scattered light intensity". In this section we present dependence of these values on some parameters we described above. 


\subsection{General case}

Below we present results of our computations for variable field parameters $\left(\Omega_{s}, \Omega_{w}, \Delta_{s}\right)$ and medium parameters $\left(\Gamma_{w}, N, d_{a}, d_{l}\right)$, Great part of our computations was made for $\Delta_{s}=0$.

We observed that the forward scattered light intensity increases like the third power of probe field intensity e.g. like the sixth power of the Rabi frequency (Fig. 2).
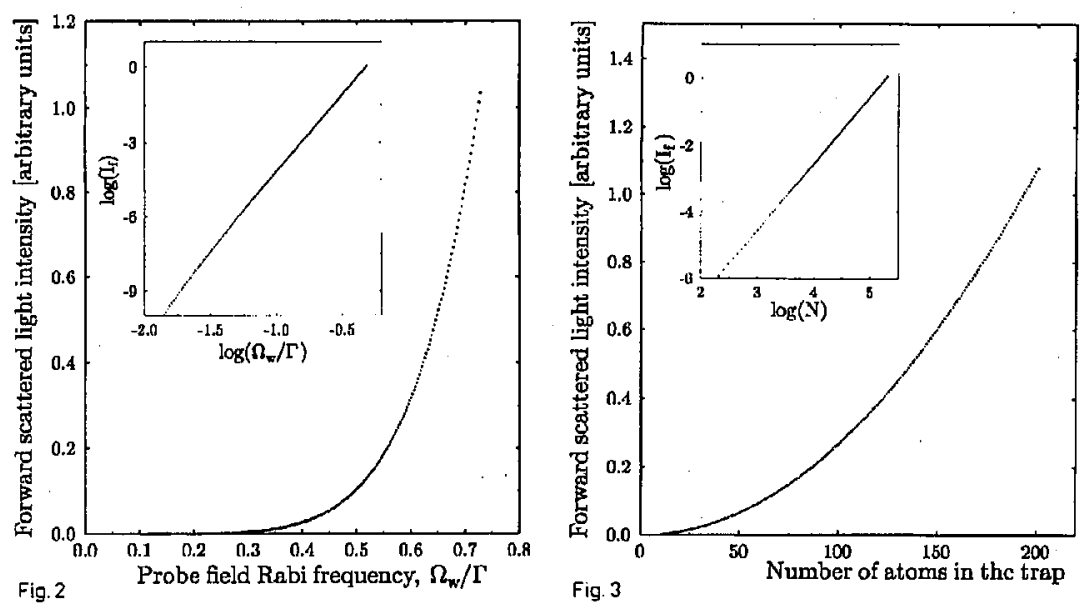

Fig. 2. Intensity of the light scattered into the forward direction $\left(I_{f}\right)$ as a function of the Rabi frequency of the probe field. Inserted log-log plot of the same quantity shows the power dependence of the intensity of the scattered light. $\Omega_{s} / \Gamma=40$.

Fig. 3. Intensity of the light scattered into forward direction $\left(I_{f}\right)$ as a function of the number of trapped atoms $(N)$. Inserted log-log plot of the same quantity shows the square dependence of the intensity of the scattered light.

We observed also that the forward scattered intensity increases like the second power of the number of trapped atoms (Fig. 3).

We examined the forward scattered light intensity as a function of the pump field detuning and we realized that it is almost Lorentz-type function but there are some minima which can be seen in the semi-logarithmic plot (Fig. 4). The case of the minima is described separately.

Forward scattered light intensity almost very weakly depends on the average distance between atoms, but the backward and total scattered light intensities have some interesting properties. The backward scattered light intensity is very small (with respect to forward scattered light intensity), but has very strong and very narrow peaks corresponding to average distances being the multiplicities of half wavelength (Fig. 5). The total scattered intensity decreases like the inversion of the average distance but has corresponding peaks. This effect is not an unexpected one as in the retarded time both directions (forward and backward) are equivalent for those values of the average distance between atoms. We examined the ratio of 

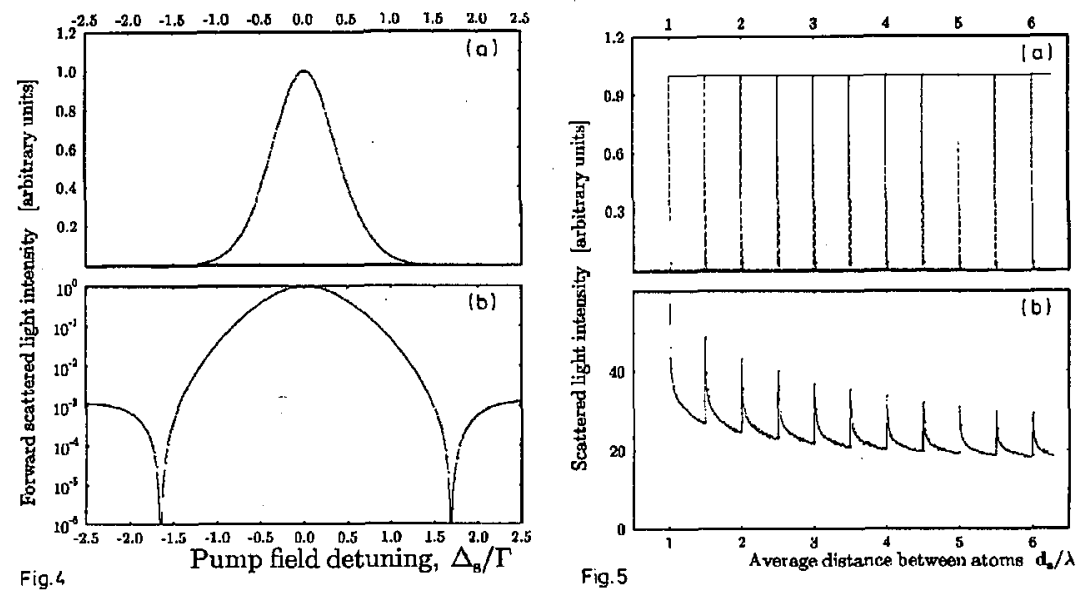

Fig. 4. (a) Forward scattered light intensity vs. pump field detuning. (b) The same quantity on the semi-logarithmic plot.

Fig. 5. (a) Forward (solid line) and backward (dashed line) scattered light intensity vs. average distance between atoms. (b) Total scattered light intensity vs. average distance between atoms.
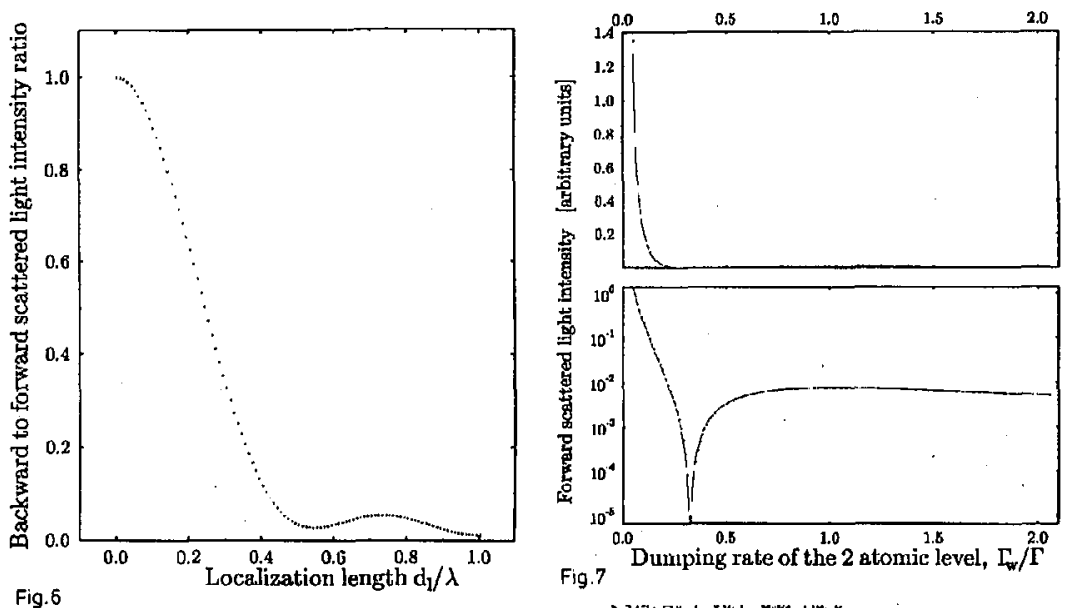

Fig.7

Fig. 6. Backward to forward scattered light intensity ratio vs. localization length.

Fig. 7. Forward scattered light intensity as a function of the medium atom state dumping rate.

the backward peak to forward scattered light intensity as a function of localization length and we show it in Fig. 6.

We also studied light scattering as a function of the dumping rate $\Gamma_{w}$. Very interesting one is the minimum similar to the one we get as a function of pump field detuning (Fig. 7). As shown in Fig. 8 the angular spectrum of scattered light corresponding to these minima has properties of the cone emission. 


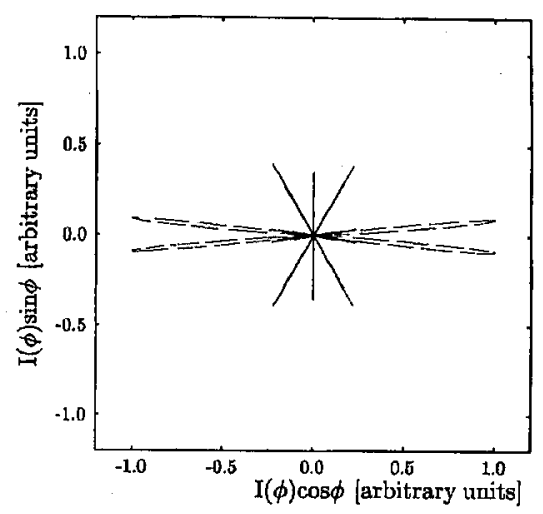

Fig. 8. Angular spectrum of the scattered light in polar coordinates.

\subsection{Cone emission case}

Below we present results corresponding to the light scattering having properties of cone emission. Cone emission has been the subject of numerous experiments and theoretical considerations in recent years [5-7], but most of them corresponds to continuous media. There are some different ideas explaining that phenomenon, but none of them seems to be fully satisfying. Our results show that mutual interaction of atoms (via the electromagnetic field) is crucial for the cone emission. Figure 9 shows angular spectra corresponding to described above minima. Qual-
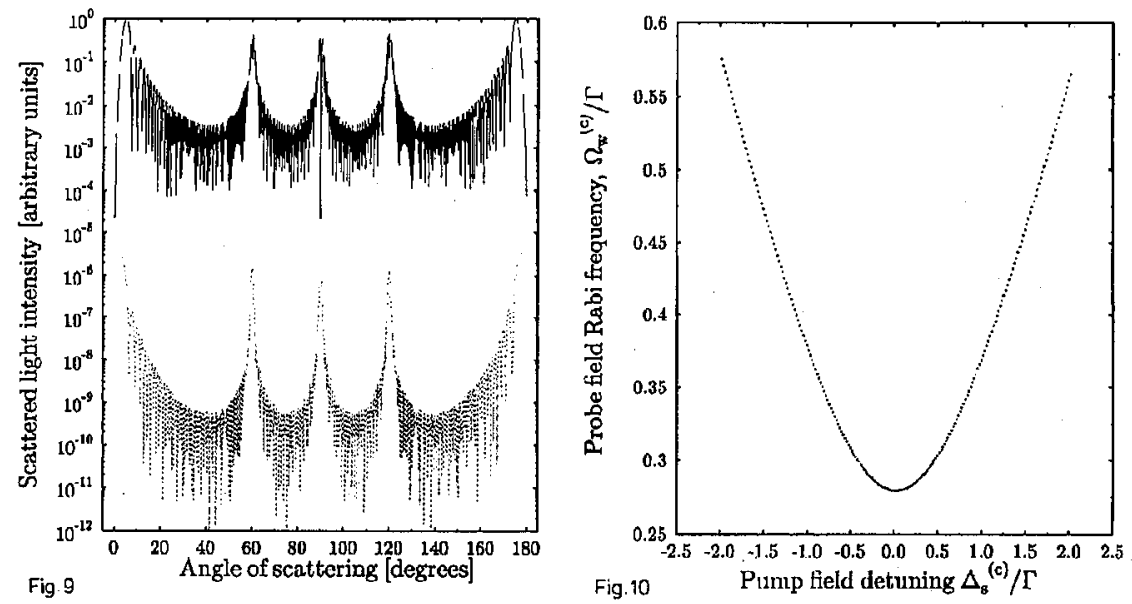

Fig. 9. Angular spectrum of the scattered light with (solid line) and without (dashed line) atom-atom interaction (semi-logarithmic plot).

Fig. 10. Dependence of the probe field Rabi frequency on the pump field detuning corresponding to cone emission for fixed damping rate. 
itative difference is that without atom-atom interaction we have not observed strong scattering into the cone near the axis of the medium (for small angles and near to $180^{\circ}$ ). We show some plots illustrating conditions of producing of the cone emission. From our point of view the most important is dependence on parameters, we cannot change them easily (in real experiment): the dumping rate (which is given and we cannot change it at all) of the medium state, number of atoms and the average distance between atoms.

As shown in Fig. 10, for fixed damping rate of the atomic level there is some threshold value of the probe field amplitude for the existence of the cone emission. We realized that changing such values as the average distance between atoms, the number of atoms, the localization length does not influence on the conditions of the cone emission producing.

\section{Conclusions}

In this paper we have investigated semi-classically the resonant light scattering on an active medium based on the three-level atom formulation. Our medium is formed by regularly distributed three-level atoms in the $V$-configuration, therefore we treat it as a discrete one. In the steady-state limit we found that there is a preferred direction of scattering, determined by the direction of the propagation of the external fields, but we also found some exceptions. For the average distance between atoms equal to the multiplicity of the wavelength of the probe field the light was scattered in the backward direction as strongly as forward.

Moreover, we realized that the cone emission from such medium is possible but without dipole-dipole interaction that phenomenon was not observed.

This work is supported by the State Committee for Scientific Research (Republic of Poland), grant number 223999203.

\section{References}

[1] C. Monroe, W. Swann, H. Robinson, C. Wieman, Phys. Rev. Lett. 65, 1571 (1990).

[2] E.L. Raab, D.E. Pritchard, V. Bagnato, C. Wieman, R. Watts, Phys. Rev. Lett. 57, 310 (1986).

[3] E.L. Raab, M.G. Prentis, A.E. Cable, S. Chu, D.E. Pritchard, Phys. Rev. Lett. 59, 2631 (1987).

[4] M. Born, E. Wolf, Principles of Optics, Pergamon Press, Oxford 1964.

[5] J.F. Valley, G. Khitrowa, H.M. Gibbs, J.W. Grantham, Xu Jiajin, Phys. Rev Lett. 64, $2362(1990)$.

[6] M.E. Crenshaw, C.D. Cantrell, Phys. Rev. A 39, 126 (1989).

[7] L. You, J. Mostowski, J. Cooper, Phys. Rev. A 46, 2906 (1992). 